\title{
COVID-19 mRNA Vaccine and Myocarditis
}

\author{
Balraj Singh ${ }^{1}$, Parminder Kaur ${ }^{2}$, Leon Cedeno ${ }^{3}$, Taulant Brahimi ${ }^{3}$, Prem Patel $^{2}$, Hartaj Virk ${ }^{2}$, Fayez Shamoon ${ }^{2}$, Manesh Bikkina ${ }^{2}$ \\ ${ }^{1}$ Department of Hematology-Oncology, Saint Joseph's University Medical Center, Paterson, New Jersey \\ ${ }^{2}$ Department of Cardiology, Saint Joseph's University Medical Center, Paterson, New Jersey \\ ${ }^{3}$ Department of Internal Medicine, Saint Joseph's University Medical Center, Paterson, New Jersey
}

How to cite this article: Singh B, Kaur P, Cedeno L, Brahimi T, Patel P, Virk H, Shamoon F, Bikkina M. COVID-10 mRNA vaccine and myocarditis. EJCRIM 2021;8: doi:10.12890/2021_002681.

Conflicts of Interests: The authors declare there are no competing interests.

This article is licensed under a Commons Attribution Non-Commercial 4.0 License

\section{ABSTRACT}

Coronavirus disease 2019 (COVID-19) is believed to have originated in the Hua nan South China Seafood Market in Wuhan and can present with a spectrum of clinical manifestations. We report the case of 24-year-old male patient who developed chest pain after administration of the second dose of the Pfizer-BioNTech mRNA COVID-19 vaccine and who was diagnosed with myocarditis on work-up.

\section{LEARNING POINTS}

- Localized injection site reactions and systemic adverse effects can occur after administration of the various COVID-19 vaccines.

- Healthcare providers should maintain a high index of suspicion regarding myocarditis after mRNA COVID-19 vaccination in the appropriate clinical scenario.

\section{KEYWORDS}

COVID-19, severe acute respiratory syndrome coronavirus 2, SARS-CoV-2, myocarditis, COVID-19 vaccine, side effects, adverse events, pericarditis, inflammation of the heart

\section{INTRODUCTION}

Coronavirus disease 2019 (COVID-19) caused by SARS-CoV-2 (severe acute respiratory syndrome coronavirus 2) has resulted in a global health and economic crisis. Initially considered a respiratory tract disease, it is now known that it can cause multiple organ dysfunction and presents with a wide variety of complications (gastrointestinal, neurological, haematological, thromboembolic, immune and cardiovascular). Complications and outcomes may depend on disease severity and the comorbidities of the infected patient. For optimal management of these patients, understanding of the various systemic manifestations and complications of SARS-CoV-2 is essential. Vaccination against SARS-CoV-2 infection is considered a valid approach for controlling the pandemic.

\section{CASE DESCRIPTION}

We describe the case of a 24-year-old Caucasian man with no past medical history who presented to the Emergency Department (ED) with chest pain which had woken him from sleep. The pain was left-sided, severe, constant, non-radiating, was associated with headache and had started 30 min before arrival in the ED. The patient denied taking any over-the-counter medications for pain relief.

The patient had received his second dose of BNT162b2 (Pfizer-BioNTech COVID-19 vaccine) 3 days before the episode. On the day he received the second dose, he developed subjective fever, chills, fatigue, headache and lower back pain, which resolved over the next 24 
hours. The night before presenting to the hospital, he had shovelled snow for about 30 minutes. The next morning, he developed chest pain which prompted him to come to the ED.

A review of symptoms was negative for nausea, vomiting, diaphoresis, dizziness, light-headedness, vision changes, orthopnoea, shortness of breath and paroxysmal nocturnal dyspnoea. The patient was born and raised in the state of New Jersey, USA and works as a maintenance custodian. He denied recent sick contacts or travel. He drinks alcohol socially and has never used tobacco products or illicit drugs such as cocaine or amphetamines. His parents are alive and healthy, and there was no family history of sudden cardiac death, other cardiac disease or autoimmune disease.

On presentation, the patient's temperature was $37.6^{\circ} \mathrm{C}$, blood pressure was $91 / 52 \mathrm{mmHg}$, heart rate was 92 beats per minute, respiratory rate was 18 breaths per minute, oxygen saturation was $98 \%$ on room air and his body mass index was $27.7 \mathrm{~kg} / \mathrm{m}^{2}$. Physical examination was unremarkable.

On laboratory evaluation, the following values were noted: haemoglobin $12.9 \mathrm{~g} / \mathrm{dl}$ (13.5-17.5 g/dl), haematocrit 38.7\% (41-53\%), total leucocyte count $7.3 \times 10^{3} / \mathrm{mm}^{3}\left(4.5-11 \times 103 / \mathrm{mm}^{3}\right)$, absolute neutrophil count $5.0 \times 10^{3} / \mathrm{mm}^{3}\left(1.3-7.8 \times 10^{3} / \mathrm{mm}^{3}\right)$, absolute lymphocyte count $1.14 \times 10^{3} / \mathrm{mm}^{3}\left(0.9-4.6 \times 10^{3} / \mathrm{mm}^{3}\right)$, platelets $161 \mathrm{~K} / \mathrm{mm}^{3}$ (140-440 K/mm $)$, MCV $89 \mathrm{fl}(80-100 \mathrm{fl})$, RDW 12.5\% (0.5-16.5\%), blood urea nitrogen $13 \mathrm{mg} / \mathrm{dl}$ (7-23 mg/dl), serum creatinine $0.83 \mathrm{mg} / \mathrm{dl}(0.60-1.30 \mathrm{mg} / \mathrm{dl})$, high-sensitivity troponin I initial value 2,529 pg/ml (3-23

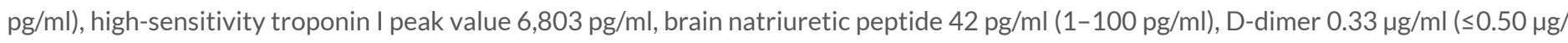
$\mathrm{ml})$, erythrocyte sedimentation rate $21 \mathrm{~mm} / \mathrm{hr}(0-10 \mathrm{~mm} / \mathrm{hr})$, C-reactive protein $24.8 \mathrm{mg} / \mathrm{l}(\leq 9.9 \mathrm{mg} / \mathrm{l})$, creatinine kinase $450 \mathrm{U} / \mathrm{l}$ (30-223 $\mathrm{U} / \mathrm{I})$, prothrombin time $14.2 \mathrm{~s}(12.2-14.9 \mathrm{~s})$, international normalized ratio 1.1 (0.9-1.1), partial thromboplastin time $30 \mathrm{~s}(21.3-35.1 \mathrm{~s})$, iron level $43 \mu \mathrm{g} / \mathrm{dl}(50-212 \mu \mathrm{g} / \mathrm{dl})$, total iron binding capacity $274 \mu \mathrm{g} / \mathrm{dl}$ (150-450 $\mu \mathrm{g} / \mathrm{dl})$, iron saturation 16\% (15-50\%), ferritin 108.0 ng/ $\mathrm{ml}(18.7-323.0 \mathrm{ng} / \mathrm{ml})$, folate $19.6 \mathrm{ng} / \mathrm{ml}(\geq 4.1 \mathrm{ng} / \mathrm{ml})$, thyroid stimulating hormone $1.415 \mathrm{mlU} / \mathrm{ml}(0.450-5.330 \mathrm{mlU} / \mathrm{ml})$, sodium $136 \mathrm{mEq} / \mathrm{l}$ (135-145 mEq/l), potassium $3.6 \mathrm{mEq} / \mathrm{l}$ (3.5-5 mEq/l), chloride $102 \mathrm{mEq} / \mathrm{l}$ (98-107 mEq/l), bicarbonate $23 \mathrm{mEq} / \mathrm{l}$ (21-31 mEq/l), glucose 93 $\mathrm{mg} / \mathrm{dl}$ (70-110 mg/dl), calcium $8.8 \mathrm{mg} / \mathrm{dl}$ (8.6-10.3 mg/dl), bilirubin $0.5 \mathrm{mg} / \mathrm{dl}(0.3-1.1 \mathrm{mg} / \mathrm{dl})$, total protein $6.9 \mathrm{~g} / \mathrm{dl}$ (6.4-8.4 g/dl), albumin $4.4 \mathrm{~g} / \mathrm{dl}$ (3.5-5.7 g/dl), alkaline phosphatase $54 \mathrm{U} / \mathrm{l}(34-104 \mathrm{U} / \mathrm{l})$, aspartate aminotransferase $22 \mathrm{U} / \mathrm{l}$ (13-39 U/I), alanine aminotransferase $9 \mathrm{U} / \mathrm{l}$ (7-52 U/l), phosphorus $3 \mathrm{mg} / \mathrm{dl}$ (2.5-5 mg/dl), magnesium $1.9 \mathrm{mg} / \mathrm{dl}$ (1.7-2.5 mg/dl), cholesterol $109 \mathrm{mg} / \mathrm{dl}$ ( $\leq 199 \mathrm{mg} / \mathrm{dl})$, triglycerides

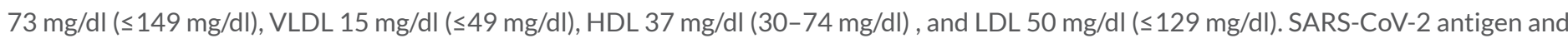
a PCR test were negative.

Coxsackievirus B1 antibody titre was 1:8 (<1:8 titre) and so essentially negative. No antibodies against coxsackievirus B2, B3, B4, B5 and B6 serotypes were detected. Notably, the patient had IgG antibodies (each positive with a titre of 1:400 where the normal value is $<1: 100$ ) against coxsackie A7, A9, A16 and A24 serotypes but was negative for IgM antibodies, suggesting past infection. Parvo B19 IgM 0.2 (0-0.8) and IgG 0.3 (0-0.8) were also negative.

The PCR panel was negative for adenovirus, human metapneumovirus, rhinovirus, enterovirus, influenza A, influenza B, parainfluenza virus 1, 2, 3 and 4, respiratory syncytial virus, Bordetella parapertussis, Bordetella pertussis, Chlamydia pneumoniae, Mycoplasma pneumoniae, HH6 and Lyme disease. HIV, Hep B and C were also negative.

Chest x-ray and CT angiography were unremarkable. An electrocardiogram (EKG) showed normal sinus rhythm, a normal axis, 76 beats per minute, and a 1-mm ST-depression in lead III (Fig. 1). An echocardiogram showed an ejection fraction of 55\%. Cardiac catheterization showed normal coronary arteries. Cardiac magnetic resonance imaging (MRI) with and without contrast showed linear sub-epicardial enhancement involving the lateral wall of the left ventricle consistent with acute myocarditis (Fig. 2). An endomyocardial biopsy was not performed due to the patient's improving symptoms and lack of ventricular arrhythmias or significant cardiac wall motion abnormalities. The patient was hospitalized for 4 days and discharged in a stable condition. He was seen in an outpatient clinic 6 weeks later, is doing well and is back at work.

\section{DISCUSSION}

Myocarditis is inflammation of the myocardium and can be caused by a variety of infectious (viruses, bacteria and protozoa) and noninfectious diseases. Causative viruses include, but are not limited, enteroviruses (especially coxsackievirus), adenovirus, parvovirus B19, human herpesvirus 6, human cytomegalovirus, HIV and hepatitis virus ${ }^{[1,2]}$. Coronaviruses (severe acute respiratory syndrome (SARS, 2002), Middle East respiratory syndrome coronavirus (MERS-CoV, 2012) and the current SARS-CoV-2) are known to cause myocarditis ${ }^{[3]}$. Myopericarditis following smallpox vaccination among vaccinia-naive US military personnel has also been reported ${ }^{[4]}$. Non-infectious causes include alcohol, cocaine, medications and systemic conditions like coeliac disease, collagen vascular disease, sarcoidosis, thyrotoxicosis, Kawasaki disease, inflammatory bowel disease, granulomatosis with polyangiitis, and hyper-eosinophilia ${ }^{[5]}$. We report the case of $24-y e a r-$ old male patient who developed chest pain after administration of his second dose of the Pfizer-BioNTech COVID-19 vaccine and who was diagnosed with myocarditis on work-up. 


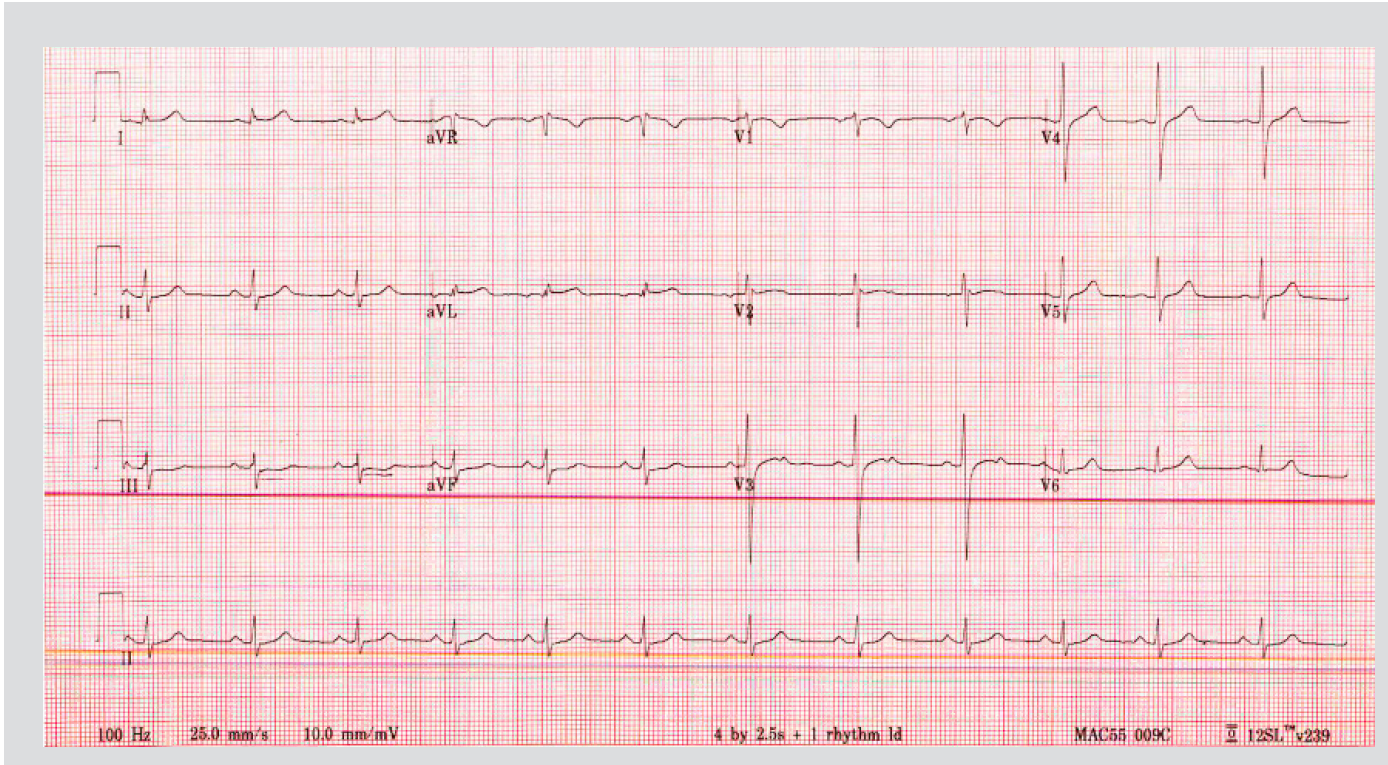

Figure 1. Electrocardiogram showing normal sinus rhythm, a normal axis, 76 beats per minute and a 1-mm ST-depression in lead III
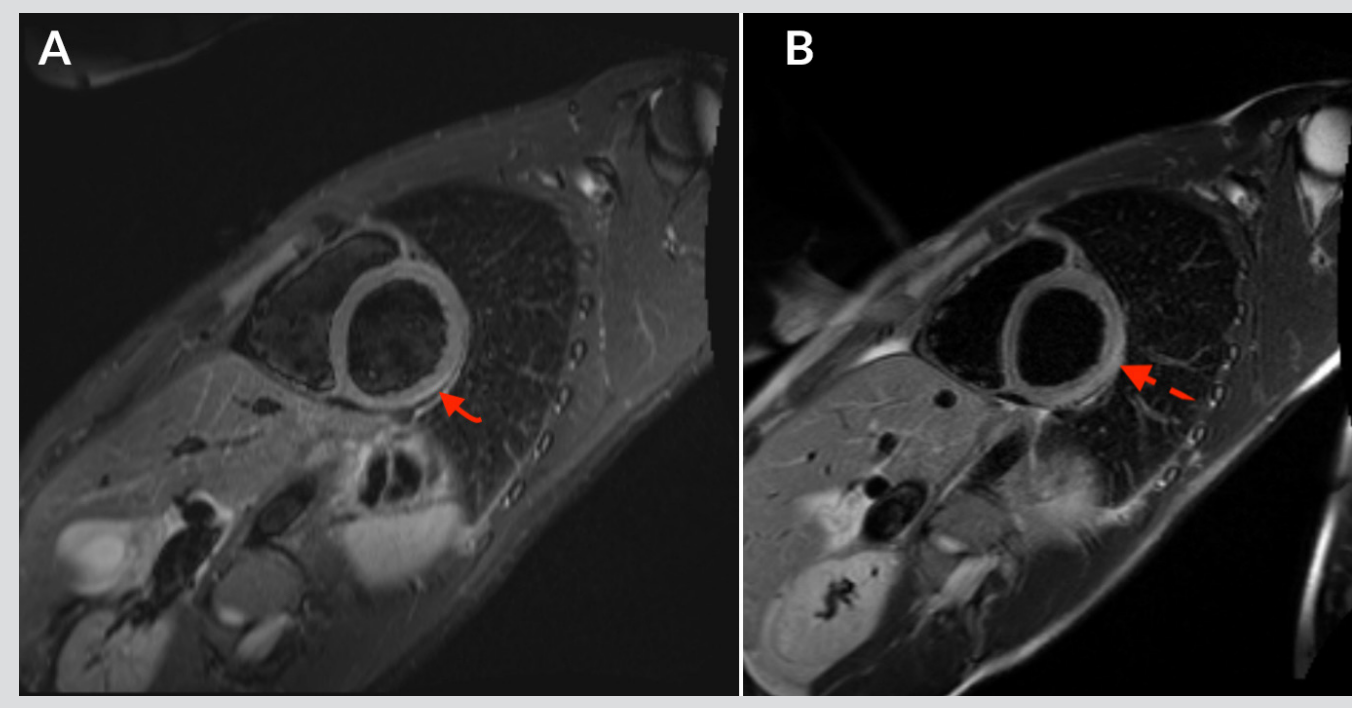

Figure 2. Cardiac magnetic resonance imaging (MRI) with and without contrast showed linear sub-epicardial enhancement involving the lateral wall of the left ventricle consistent with acute myocarditis: STIR image (panel A) and T2 image (panel B)

Vaccines cause the immune system to elicit neutralizing antibodies that provide protection against infection. Different technology platforms (RNA vaccines, replication-incompetent vector vaccines, recombinant protein vaccines and inactivated vaccines) are being used to develop vaccines against COVID-19 infection. In the United States, two COVID-19 mRNA vaccines (BNT162b2 (Pfizer-BioNTech) and mRNA-1273 (Moderna)) and one COVID-19 adenovirus vector vaccine (Ad26.COV2.S (Janssen)) have been given emergency use authorization. Localized injection site reactions and systemic adverse effects can occur after the administration of the different COVID-19 vaccines. Systemic events include fatigue, fever, chills, headache and myalgias. Serious adverse effects like anaphylaxis have been reported after Pfizer-BioNTech and Moderna COVID-19 vaccine administration ${ }^{[6]}$. Thrombocytopenia and thrombotic events (cerebral venous thrombosis, splanchnic vein thrombosis, pulmonary embolism) have developed after vaccination with the AstraZeneca vaccine (ChAdOx1 nCov-19, a recombinant adenoviral vector encoding the spike protein antigen of SARS-CoV-2) ${ }^{[7]}$.

An increasing number of cases of myocarditis and pericarditis after mRNA COVID-19 vaccination have been reported in the Vaccine Adverse Event Reporting System (VAERS). Most cases are in male adolescents, typically within several days of COVID-19 vaccination and more commonly after the second dose ${ }^{[8]}$. In Israel, Mevorach et al. identified 110 cases of myocarditis among 5 million people who were administered two doses of the Pfizer-BioNTech vaccine in the month before their myocarditis diagnosis and $90 \%$ of cases were in men. Among the vaccinated young men, the rate was 5-25 times the background rate for myocarditis [9]. Our patient was also young (24 years old), presented with chest pain 3 days after receiving his second dose of the Pfizer-BioNTech mRNA COVID-19 vaccine, and was diagnosed with myocarditis. Extensive work-up was done to exclude other possible causes of myocarditis in our patient. Healthcare providers should maintain a high index of suspicion regarding myocarditis after COVID-19 vaccination in the appropriate clinical situation. 
We report the case of 24-year-old male patient who developed chest pain after receiving the second dose of the Pfizer-BioNTech mRNA COVID-19 vaccine and who was diagnosed with myocarditis. Healthcare providers should maintain a high index of suspicion regarding myocarditis after COVID-19 vaccination in the appropriate clinical situation. We emphasize that the benefits of vaccination far outweigh the risk given COVID-19 infection can cause multisystem organ dysfunction leading to morbidity and mortality and given that reported cases of myocarditis are mild and rare and most patients recover. Vaccination is a vital, very effective and life-saving measure against COVID-19 infection.

\section{REFERENCES}

1. Bowles NE, Ni J, Kearney DL, Pauschinger M, Schultheiss H-P, McCarthy R, et al. Detection of viruses in myocardial tissues by polymerase chain reaction: evidence of adenovirus as a common cause of myocarditis in children and adults. J Am Coll Cardiol 2003;42(3):466-472.

2. Rose NR. Viral myocarditis. Curr Opin Rheumatol 2016;28(4):383-389.

3. Rezkalla SH, Kloner RA. Viral myocarditis: 1917-2020: from the influenza A to the COVID-19 pandemics. Trends Cardiovasc Med 2021;31(3):163-169.

4. Halsell JS, Riddle JR, Atwood JE, Gardner P, Shope R, Poland GA, et al. Myopericarditis following smallpox vaccination among vaccinia-naive US military personnel. JAMA 2003;289(24):3283-3289.

5. Veronese G, Ammirati E, Cipriani M, Frigerio M. Fulminant myocarditis: characteristics, treatment, and outcomes. Anatol J Cardiol 2018;19(4):279-286.

6. Shimabukuro TT, Cole M, Su JR. Reports of anaphylaxis after receipt of mRNA COVID-19 vaccines in the US-December 14, 2020-January 18, 2021.JAMA 2021;325(11):11011102.

7. Greinacher A, Thiele T, Warkentin TE, Weisser K, Kyrle PA, Eichinger S. Thrombotic thrombocytopenia after ChAdOx1 nCov-19 vaccination. NEngI J Med 2021;384(22):20922101.

8. Centers for Disease Control and Prevention. Myocarditis and pericarditis following mRNA COVID-19 vaccination. 2021. Available from: https://www.cdc.gov/ coronavirus/2019-ncov/vaccines/safety/myocarditis.html.

9. Vogel G, Couzin-Frankel J. Israel reports link between rare cases of heart inflammation and COVID-19 vaccination in young men. Science 2021. Available from: https://www. sciencemag.org/news/2021/06/israel-reports-link-between-rare-cases-heart-inflammation-and-covid-19-vaccination 